\title{
CORRECTION
}

\section{Correction to: TGF-betal gene-modified, immature dendritic cells delay the development of inflammatory bowel disease by inducing $\mathrm{CD} 4(+)$ Foxp3(+) regulatory $\mathrm{T}$ cells}

Zhijian Cai (D), Wei Zhang, Min Li, Yinpu Yue, Fei Yang, Lei Yu, Xuetao Cao and Jianli Wang

Cellular \& Molecular Immunology (2020) 17:190-191; https://doi.org/10.1038/s41423-019-0338-6

Correction to: Cellular \& Molecular Immunology https://doi.org/ 10.1038/cmi.2009.107, published online 18 January 2010

Zhijian Cai, Wei Zhang, Min Li, Yinpu Yue, Fei Yang, Lei Yu, Xuetao Cao \& Jianli Wang TGF- $\beta 1$ gene-modified, immature dendritic cells delay the development of inflammatory bowel disease by inducing $\mathrm{CD}^{+}{ }^{+} \mathrm{Foxp}^{+}{ }^{+}$regulatory T cells. Cell Mol Immunol. 2010 Jan;7(1):35-43.
In the published version of Fig. 5b, the data of the "ISOTYPE" and "Control-DC" groups were mistakenly presented with incorrected images. The results from another independent experiment are presented to replace the published ones. The corrected version of Fig. 5 is shown below.

Although we regret our mistake during figure assembly and would like to apologize for any inconvenience it may have caused, we did not manipulate our data in any way. This unintentional error also has no bearing on the work's scientific conclusions. 
a
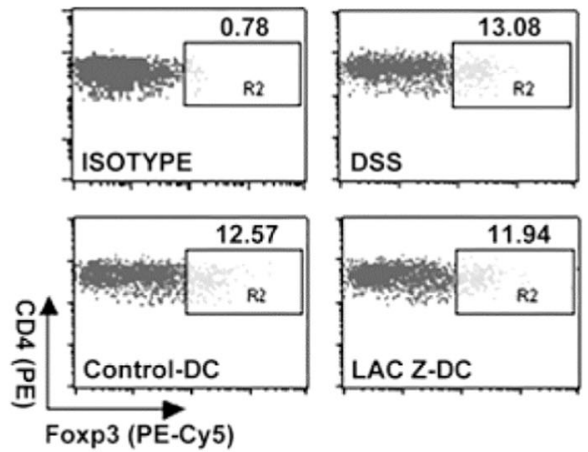

b
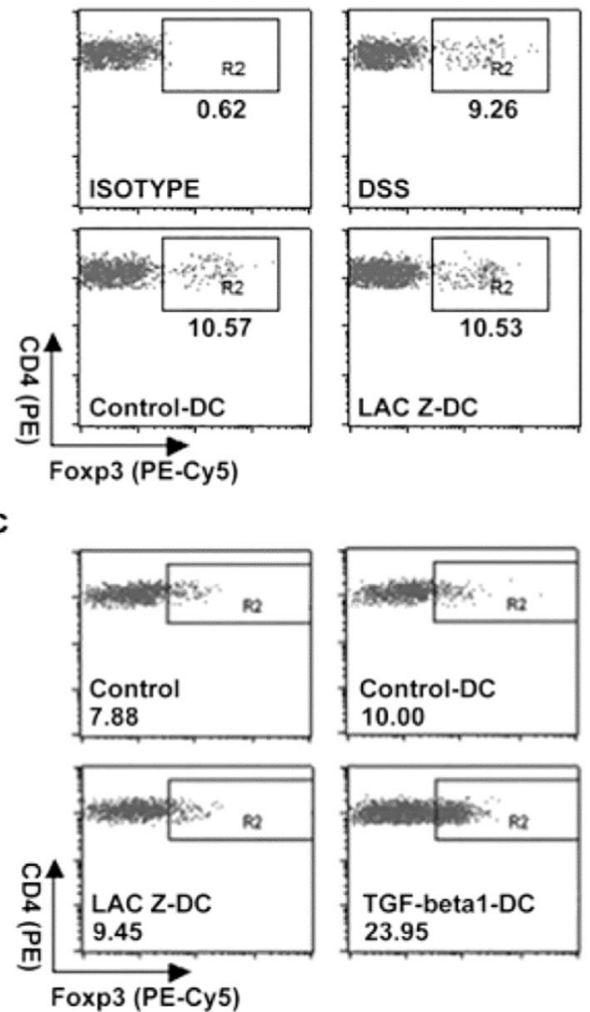

23.95
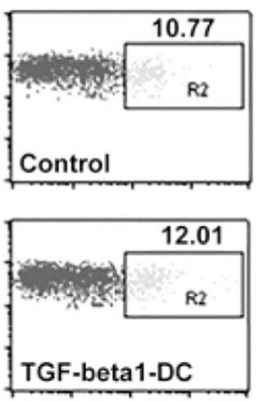

TGF-beta1-DC
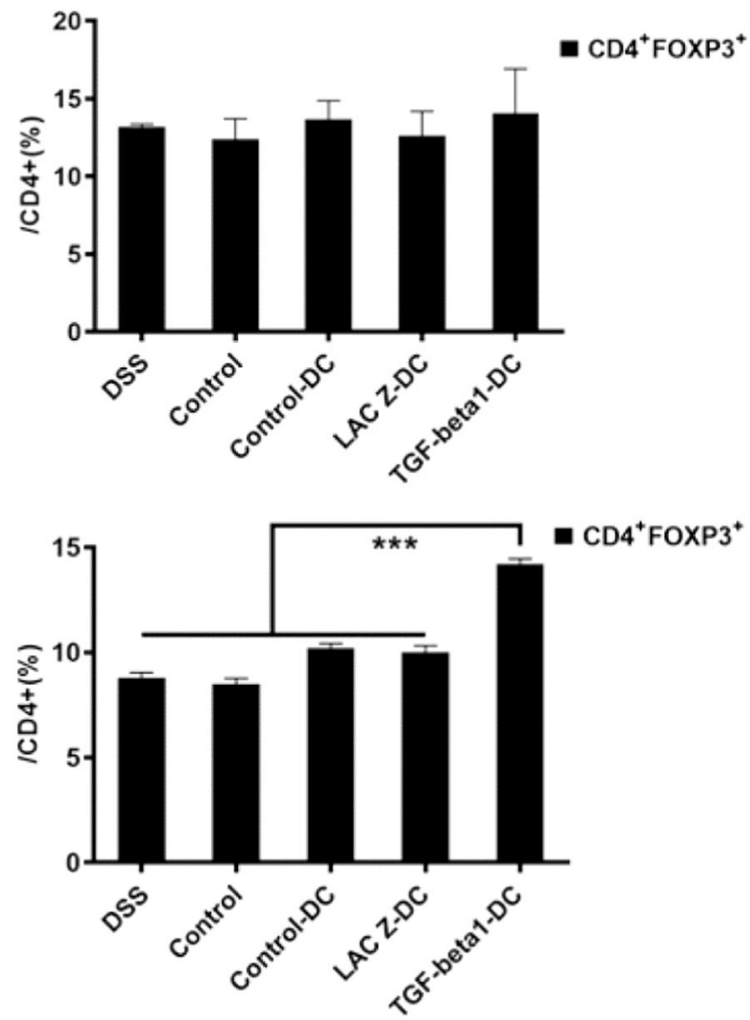

TGF-beta1-DC
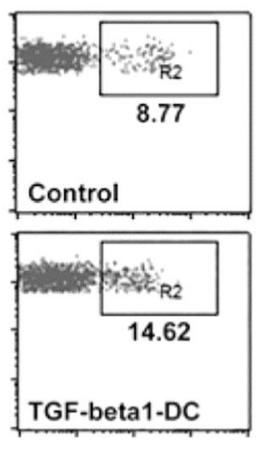

$\mathrm{CD}^{+} \mathrm{FOXP}^{+}$
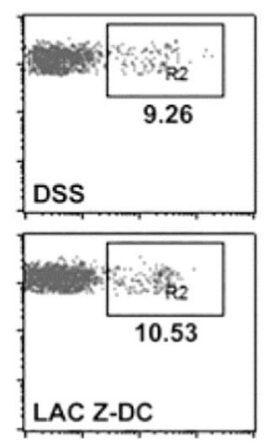

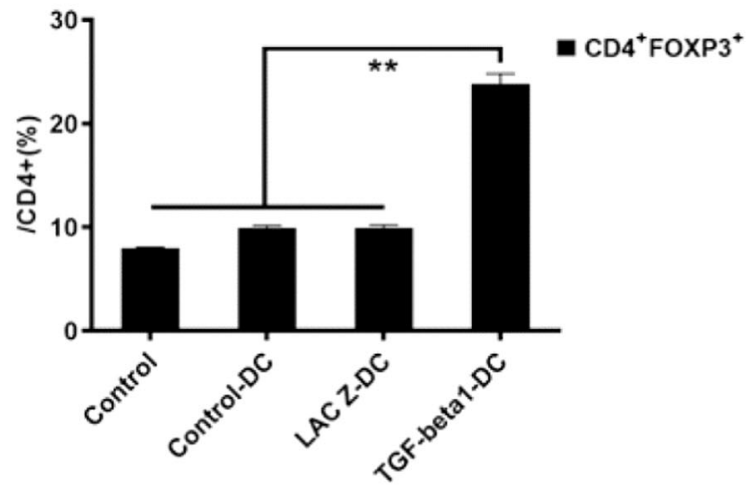

Fig. 5 TGF- $\beta 1-D C s$ can increase the relative numbers of $\mathbf{C D 4}{ }^{+}$Foxp $3^{+}$Tregs in vivo and in vitro. a The relative CD4 ${ }^{+}$Foxp $3^{+}$Treg numbers from spleens of each group mice. $\mathbf{b}$ The relative $C D 4^{+}$Foxp $3^{+}$Treg numbers from $\mathrm{mLNs}$ of each group mice. CD4 ${ }^{+}$cells were gated and analyzed for Foxp $3^{+}$Tregs. c TGF- $\beta 1-D C s$ can increase the relative numbers of CD4 ${ }^{+}$Foxp3 ${ }^{+}$Tregs in vitro. ${ }^{*} P<0.05$, ${ }^{* *} P<0.01$; data are representative of three independent experiments $(n=3)$. DC, dendritic cell; TGF- $\beta 1$, transforming growth factor- $\beta 1$. 